\title{
Outage Probability Analysis of Distributed Reception with Hard Decision Exchanges
}

\author{
Rui Wang, D. Richard Brown III, Min Ni \\ Dept. of Electrical and Computer Eng. \\ Worcester Polytechnic Institute \\ 100 Institute Rd, Worcester, MA 01609 \\ Email: \{rwang,drb,minni@wpi.edu\}
}

\author{
Upamanyu Madhow \\ Dept. of Electrical and Computer Eng. \\ University of California Santa Barbara \\ CA 93106 \\ Email: madhow@ece.ucsb.edu
}

\author{
Patrick Bidigare \\ Raytheon BBN Technologies \\ 1300 17th St N Suite 400 \\ Arlington, VA 22209 \\ Email: bidigare@ieee.org
}

\begin{abstract}
This paper considers the problem of jointly decoding binary messages from a single distant transmitter to a cooperative receive cluster. The nodes in the receive cluster exchange information to decode messages from the transmitter. The outage probability of distributed reception with binary hard decision exchanges is compared with the outage probability of ideal receive beamforming with unquantized observation exchanges. Low-dimensional analysis and numerical results show, via two simple but surprisingly good approximations, that the outage probability performance of distributed reception with hard decision exchanges is well-predicted by the SNR of ideal receive beamforming after subtracting a hard decision penalty of slightly less than $2 \mathrm{~dB}$. These results, developed in non-asymptotic regimes, are consistent with prior asymptotic results (for a large number of nodes and low per-node SNR) on hard decisions in binary communication systems.
\end{abstract}

Index Terms-distributed reception, cooperative communications, beamforming, outage probability

\section{INTRODUCTION}

We consider the distributed reception scenario in Fig. 1 with a single distant transmitter and a cluster of $k$ receive nodes. The goal is to communicate messages over the forward link from the distant transmitter to all of the receive nodes. The receive nodes form a fully-connected network and can reliably exchange information to jointly decode the messages from the distant transmitter, i.e., the receive cluster can perform distributed reception.

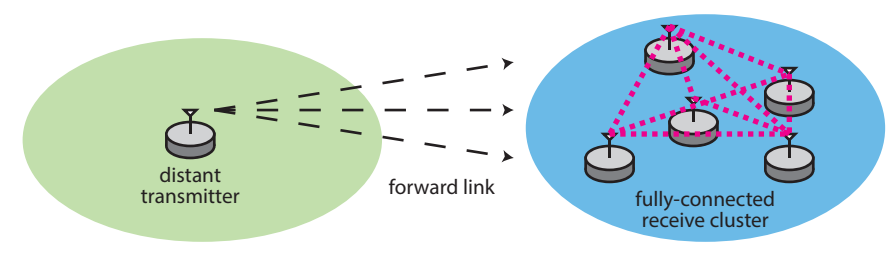

Fig. 1. Distributed reception scenario.

Recent information theoretic studies [1]-[4] have shown that distributed reception techniques have potential to increase diversity, improve capacity, and improve interference rejection, even with tight network throughput constraints. Several techniques have been proposed to achieve these gains including

This work was supported by the National Science Foundation awards CCF1302104 and CCF-1319458. link-layer iterative cooperation [5], [6], distributed iterative receiver message-passing [7], and most-reliable/least-reliable bit exchange iterative decoding [8]-[13]. A limitation of all of these techniques is that they are based on iterative transmissions and decoding. As such, the backhaul requirements are variable and the decoding latency can be significant if the number of iterations is large.

A non-iterative distributed reception technique was recently considered in [14] for the case of a binary modulated forward link. Unlike the most-reliable/least-reliable bit exchange techniques in which information is transmitted over the network based on requests from other receivers, the approach in [14] is for some or all of the receive nodes to quantize each demodulated bit (prior to decoding) and then broadcast all of these quantized values to the other receivers in the cluster. The locally unquantized information at each receive node is then combined with the quantized information from other receive nodes for subsequent decoding. Numerical results showed that the outage probability penalty of exchanging binary hard decisions rather than unquantized observations (ideal receive beamforming) was less than $1.5 \mathrm{~dB}$ in the cases considered.

In this paper we analyze the outage probability of distributed reception with hard decision exchanges in the case of a binary modulated forward link and independent and identically distributed Rayleigh fading forward link channels. Unlike [14] where locally unquantized information at each receive node is combined with the quantized information from other receive nodes, we make the simplifying assumption that all observations are either quantized (distributed reception with hard decision exchanges) or unquantized (ideal receive beamforming). The performance of ideal receiver beamforming depends only on the norm of the vector channel from the transmitter to the receivers, with outage occurring when this norm falls below a threshold corresponding to the particular coded modulation strategy used. While a closed-form expression for the outage probability of distributed reception with hard decision exchanges appears intractable, low-dimensional analysis and numerical results lead to simple yet accurate approximations that depend only on the norm of the vector channel. Thus, the performance of distributed reception with hard decision exchanges tracks that of ideal receive beamforming, except for a hard decision penalty. This penalty is slightly less than 
$2 \mathrm{~dB}$ in the cases considered, consistent with prior results on the penalty of hard decisions in binary communication systems [15]-[17].

\section{SySTEM MODEL}

The forward link complex channel from the distant transmitter to receive node $i$ is denoted as $g_{i}$ for $i=1, \ldots, k$ and the vector channel is denoted as $\boldsymbol{g}=\left[g_{1}, \ldots, g_{k}\right]^{\top}$. Given a realvalued channel input $\sqrt{\mathcal{E}_{s}} X$ with $\mathrm{E}[X]=0$, var $[X]=1$ and $\mathcal{E}_{s}$ denoting the energy per forward link symbol, the phasecorrected signal at the $i^{\text {th }}$ receive node can be written as

$$
Y_{i}=h_{i} X+W_{i}
$$

where $h_{i}:=\sqrt{2\left|g_{i}\right|^{2} \mathcal{E}_{s} / N_{0}}, N_{0} / 2$ is the additive white Gaussian noise power spectral density, and $W_{i} \sim \mathcal{N}(0,1)$. The quantity $h_{i}^{2}$ corresponds to the signal-to-noise ratio (SNR) of the forward link symbols at receive node $i$. For notational convenience, we define the parameter $\alpha:=\sqrt{2 \mathcal{E}_{s} / N_{0}}$ and note that $h_{i}=\alpha\left|g_{i}\right|$. We also denote the scaled channel magnitude vector $\boldsymbol{h}=\left[h_{1}, \ldots, h_{k}\right]^{\top}$.

We assume:

1) The noise realizations are spatially and temporally independent and identically distributed (i.i.d.)

2) The forward link complex channel $g_{i}$ is constant within a symbol duration and is spatially and temporally i.i.d.

3) The magnitude of each complex channel $\left|g_{i}\right|$ follows the $\operatorname{Rayleigh}(\sigma)$ distribution with $\sigma^{2}=0.5$.

The receivers can reliably exchange information to jointly decode $X$. We assume that each receive node quantizes its observation by making a hard decision on the transmitted symbol and then broadcasts this hard decision over the local area network to the other receive nodes. Let $Z_{i}=\mathcal{Q}_{i}\left(Y_{i}\right)$ where $\mathcal{Q}_{i}(\cdot)$ represents the quantizer at receive node $i$ and further denote the vector channel output $\boldsymbol{Z}=\left[Z_{1}, \ldots, Z_{k}\right]^{\top}$. Since communication among the receive nodes is reliable, all receive nodes know $\boldsymbol{Z}$. We consider the outage probability

$$
p_{\text {out }}=\operatorname{Prob}\left(I_{\boldsymbol{h}}(X ; \boldsymbol{Z})<r_{\text {out }}\right)
$$

where $r_{\text {out }}$ is the outage rate and $I_{\boldsymbol{h}}(X ; \boldsymbol{Z})$ is the mutual information of the channel $X \rightarrow Z$ given the scaled channel magnitudes $\boldsymbol{h}$. Our focus in this paper is on a setting with equiprobable binary channel inputs $X$ and two different receive strategies: (i) ideal receive beamforming with $Z_{i}=Y_{i}$ for all $i=1, \ldots, k$ and (ii) distributed reception with hard decision exchanges such that $Z_{i}=\operatorname{sign}\left(Y_{i}\right)$ for all $i=1, \ldots, k$. Since ideal receive beamforming is optimal, it is of interest to quantify the performance loss of distributed reception with hard decision exchanges with respect to ideal receive beamforming.

\section{OUTAGE PROBABILITY ANALYSIS}

In this section, we analyze the outage probability of ideal receive beamforming (distributed reception with unquantized observation exchanges) and distributed reception with binary hard decision exchanges.

\section{A. Preliminaries}

We first state a well-known result that is used in the following sections. For $\left|g_{i}\right|$ i.i.d. Rayleigh $(\sigma)$ distributed with $\sigma^{2}=0.5,\|\boldsymbol{g}\|^{2} \sim \Gamma(k, 1)$. Thus, $\|\boldsymbol{h}\|^{2} \sim \Gamma\left(k, \alpha^{2}\right)$. If we define the $k$-dimensional quadrant

$$
\mathcal{H}(c):=\left\{\boldsymbol{h} \in \mathbb{R}^{k}:\|\boldsymbol{h}\|^{2}<c^{2} \text { and } h_{i} \geq 0 \forall i\right\}
$$

we can write the probability $p_{k}(\alpha, c):=\operatorname{Prob}(\boldsymbol{h} \in \mathcal{H}(c))$ as

$$
\begin{aligned}
p_{k}(\alpha, c) & =\operatorname{Prob}\left(\|\boldsymbol{h}\|^{2}<c^{2}\right) \\
& =\int_{0}^{c^{2}} f_{\|\boldsymbol{h}\|^{2}}(u) d u \\
& =F_{\|\boldsymbol{h}\|^{2}}\left(c^{2}\right) \\
& =1-\sum_{i=0}^{k-1} \frac{1}{i !}\left(\frac{c^{2}}{\alpha^{2}}\right)^{i} \exp \left[-\frac{c^{2}}{\alpha^{2}}\right]
\end{aligned}
$$

where $f_{\|\boldsymbol{h}\|^{2}}()$ and $F_{\|\boldsymbol{h}\|^{2}}()$ denote the probability density function (pdf) and cumulative distribution function (cdf) of the Gamma-distributed random variable $\|\boldsymbol{h}\|^{2}$, respectively. As observed in the following section, the outage probability of ideal receive beamforming can be exactly expressed as $p_{k}(\alpha, c)$ with an appropriately chosen quadrant radius $c$.

\section{B. Ideal Receive Beamforming}

Given an unquantized observation vector $\boldsymbol{Y}=$ $\left[Y_{1}, \ldots, Y_{k}\right]^{\top}$ with $Y_{i}$ defined in (1), the ideal receive beamformer computes the scalar channel output

$$
Z=\left[\begin{array}{lll}
h_{1} & \ldots & h_{k}
\end{array}\right] \boldsymbol{Y}=\|\boldsymbol{h}\|^{2} X+\tilde{W}
$$

where $\tilde{W} \sim \mathcal{N}\left(0,\|\boldsymbol{h}\|^{2}\right)$. When $X= \pm 1$ equiprobably, the mutual information of this channel is given as [18]

$$
I_{\boldsymbol{h}}(X ; Z)=\frac{1}{2} J(\|\boldsymbol{h}\|)+\frac{1}{2} J(-\|\boldsymbol{h}\|)
$$

with

$$
J(x):=\int_{-\infty}^{\infty} \frac{1}{\sqrt{2 \pi}} e^{\frac{-(u-x)^{2}}{2}} \log _{2}\left(\frac{2}{1+e^{-2 u x}}\right) d u .
$$

Note that (4) is exact but must be evaluated numerically. For $0 \leq r_{\text {out }}<1$,

$$
B\left(r_{\text {out }}\right)=\left\{\|\boldsymbol{h}\|: I_{\boldsymbol{h}}(X ; Z)=r_{\text {out }}\right\}
$$

has a unique solution due to the strict monotonicity of $I_{\boldsymbol{h}}(X ; Z)$ as a function of $\|\boldsymbol{h}\|$. The outage probability of the binary-input ideal receive beamforming channel then follows from (3) as

$$
p_{\text {out }}^{\mathrm{b} \rightarrow \mathrm{bf}}=p_{k}\left(\alpha, B\left(r_{\text {out }}\right)\right)
$$

where $\alpha=\sqrt{2 \mathcal{E}_{s} / N_{0}}$.

One difficulty with (6) is that $B\left(r_{\text {out }}\right)$ must be computed implicitly in (5). An explicit upper bound on the mutual information (and hence a lower bound on the outage probability) can be derived by relaxing the binary assumption on $X$ and allowing $X$ to be a Gaussian random variable. The mutual information in this case is

$$
I_{\boldsymbol{h}}(X ; \boldsymbol{Z})=0.5 \log _{2}\left(1+\|\boldsymbol{h}\|^{2}\right) .
$$


Fixing the outage rate $r_{\text {out }} \geq 0$, the strict monotonicity of $I_{\boldsymbol{h}}(X ; Z)$ implies that an outage occurs if and only if $\|\boldsymbol{h}\|<$ $A\left(r_{\text {out }}\right)$ with

$$
A\left(r_{\text {out }}\right)=\sqrt{2^{2 r_{\text {out }}}-1} .
$$

From (3), the outage probability of the Gaussian-input ideal receive beamforming channel then follows as

$$
p_{\text {out }}^{\mathrm{g} \rightarrow \mathrm{bf}}=p_{k}\left(\alpha, A\left(r_{\text {out }}\right)\right)
$$

Note that $A\left(r_{\text {out }}\right)<B\left(r_{\text {out }}\right)$, hence $p_{\text {out }}^{\mathrm{g} \rightarrow \mathrm{bf}}<p_{\text {out }}^{\mathrm{b} \rightarrow \mathrm{bf}}$. As shown in Section IV, however, $p_{\text {out }}^{\mathrm{g} \rightarrow \mathrm{bf}} \approx p_{\text {out }}^{\mathrm{b} \rightarrow \mathrm{bf}}$ for values of $r_{\text {out }}$ not too close to one. Hence, (8) can be considered a convenient approximation for (6) in this regime.

\section{Distributed Reception with Hard Decision Exchanges}

This section analyzes the outage probability of distributed reception with binary hard decision exchanges. Unlike ideal receive beamforming, the outage region $\mathcal{H}_{k}\left(r_{\text {out }}\right)$ of $k$ receiver distributed reception with hard decision exchanges for $k \geq 2$ receive nodes is not a simple quadrant as defined in (2). Nevertheless, based on low-dimensional analysis and numerical results with normalized channels, we observe that the dominant impact on performance is from the channel norm $\|\boldsymbol{h}\|$. Thus, we propose two radii, $C\left(r_{\text {out }}\right)$ and $D\left(r_{\text {out }}\right)$, with $B\left(r_{\text {out }}\right)<C\left(r_{\text {out }}\right)<D\left(r_{\text {out }}\right)$, such that the outage probability $p_{\text {out }}^{\mathrm{b} \rightarrow \text { hd }} \approx p_{k}\left(\alpha, C\left(r_{\text {out }}\right)\right) \approx p_{k}\left(\alpha, D\left(r_{\text {out }}\right)\right)$ with $p_{\text {out }}^{\mathrm{b} \rightarrow \text { hd }}=\operatorname{Prob}\left(I_{\boldsymbol{h}}(X ; \boldsymbol{Z})<r_{\text {out }}\right)$. We then use the results in Section III-A to compute approximations on the outage probability of distributed reception with hard decision exchanges.

1) Two Receive Nodes: In the case with two receive nodes and binary channel inputs, we can write the mutual information of the $2 \times 4$ discrete memoryless channel as

$$
\begin{aligned}
I_{\boldsymbol{h}}(X ; \boldsymbol{Z}) & =1-q_{1} q_{2} \log _{2}\left[\frac{p_{1} p_{2}}{q_{1} q_{2}}+1\right]-p_{1} q_{2} \log _{2}\left[\frac{q_{1} p_{2}}{p_{1} q_{2}}+1\right] \\
& -q_{1} p_{2} \log _{2}\left[\frac{p_{1} q_{2}}{q_{1} p_{2}}+1\right]-p_{1} p_{2} \log _{2}\left[\frac{q_{1} q_{2}}{p_{1} p_{2}}+1\right]
\end{aligned}
$$

where $p_{i}=Q\left(h_{i}\right)$ and $q_{i}=1-p_{i}$ for $i=1,2$. Denoting $\boldsymbol{p}=\left[p_{1}, p_{2}\right]^{\top}$ and given an outage rate $0<r_{\text {out }}<1$, we have

$$
\begin{aligned}
I_{\boldsymbol{h}}(X ; \boldsymbol{Z})<r_{\text {out }} & \Leftrightarrow \boldsymbol{p} \in \mathcal{P}_{2}\left(r_{\text {out }}\right) \\
& \Leftrightarrow \boldsymbol{h} \in \mathcal{H}_{2}\left(r_{\text {out }}\right)
\end{aligned}
$$

where $\mathcal{P}_{2}\left(r_{\text {out }}\right) \subset[0,0.5]^{2}$ is the set of channel transition probabilities that result in outage and $\mathcal{H}_{2}\left(r_{\text {out }}\right) \subset[0, \infty)^{2}$ is the set of scaled channel magnitudes that result in outage. The boundary of $\mathcal{H}_{2}\left(r_{\text {out }}\right)$ is plotted in Fig. 2 for the case $r_{\text {out }}=0.5$. Note that $\mathcal{H}_{2}\left(r_{\text {out }}\right)$ is not a simple quadrant as defined in (2). The boundaries of two quadrants $\mathcal{H}\left(C\left(r_{\text {out }}\right)\right)$ and $\mathcal{H}\left(D\left(r_{\text {out }}\right)\right)$ are also plotted in Fig. 2. The radii of the inner and outer quadrants were selected to match the boundary of $\mathcal{H}_{2}\left(r_{\text {out }}\right)$ at the points $\boldsymbol{h}=\left[0, C\left(r_{\text {out }}\right)\right]^{\top}$ and $\boldsymbol{h}=\frac{D\left(r_{\text {out }}\right)}{\sqrt{2}}[1,1]^{\top}$, respectively. To compute $C\left(r_{\text {out }}\right)$, one can perform the following steps:

1) Set $p_{2}=0.5$ or, equivalently, $h_{2}=0$.

2) Solve $I_{\boldsymbol{h}}(X ; \boldsymbol{Z})=r_{\text {out }}$ to determine $p_{1}$ or $h_{1}=$ $Q^{-1}\left(p_{1}\right)$.
3) Compute $C\left(r_{\text {out }}\right)=Q^{-1}\left(p_{1}\right)=h_{1}$.

To compute $D\left(r_{\text {out }}\right)$, one can perform the following steps:

1) Set $p_{1}=p_{2}=p$ or, equivalently, $h_{1}=h_{2}=h$.

2) Solve $I_{\boldsymbol{h}}(X ; \boldsymbol{Z})=r_{\text {out }}$ to determine $p$ or $h=Q^{-1}(p)$.

3) Compute $D\left(r_{\text {out }}\right)=\sqrt{2} Q^{-1}(p)=\sqrt{2} h$.

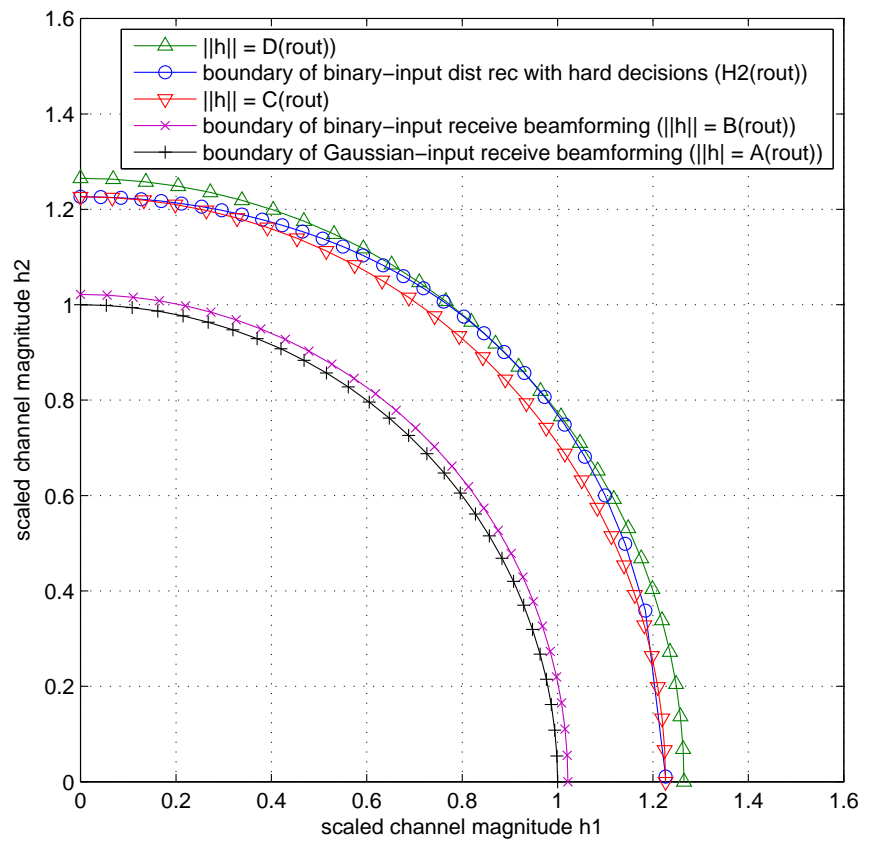

Fig. 2. Outage regions and inner/outer quadrants for the two-receiver case with $r_{\text {out }}=0.5$.

From Fig. 2, it appears that $\mathcal{H}\left(C\left(r_{\text {out }}\right)\right) \subseteq \mathcal{H}_{2}\left(r_{\text {out }}\right) \subseteq$ $\mathcal{H}\left(D\left(r_{\text {out }}\right)\right)$ in the two-receiver case, hence it is tempting to claim that $p_{2}\left(\alpha, C\left(r_{\text {out }}\right)\right)$ and $p_{2}\left(\alpha, D\left(r_{\text {out }}\right)\right)$ could serve as lower and upper bounds, respectively, for the actual outage probability $p_{\text {out }}^{\text {b } \rightarrow \text { hd }}$. A proof of this claim appears to be difficult, however, even for $k=2$ receivers. Hence, we only claim

$$
p_{\text {out }}^{\mathrm{b} \rightarrow \text { hd }} \approx p_{2}\left(\alpha, C\left(r_{\text {out }}\right)\right) \approx p_{2}\left(\alpha, D\left(r_{\text {out }}\right)\right)
$$

for $k=2$ receivers with $p_{\text {out }}^{\mathrm{b} \rightarrow \text { hd }}=\operatorname{Prob}\left(I_{\boldsymbol{h}}(X ; \boldsymbol{Z})<r_{\text {out }}\right)$ and $p_{k}(\alpha, c)$ defined in (3).

2) $k$ Receive Nodes: These approximations extend immediately to the general setting of $k$ receive nodes. We first specify two quadrants $\mathcal{H}\left(C\left(r_{\text {out }}\right)\right)$ and $\mathcal{H}\left(D\left(r_{\text {out }}\right)\right)$ with $C\left(r_{\text {out }}\right)$ computed via the following steps:

1) Set $p_{2}=\cdots=p_{k}=0.5$ or, equivalently, $h_{2}=\cdots=$ $h_{k}=0$.

2) Solve $I_{\boldsymbol{h}}(X ; \boldsymbol{Z})=r_{\text {out }}$ to determine $p_{1}$ or $h_{1}=$ $Q^{-1}\left(p_{1}\right)$.

3) Compute $C\left(r_{\text {out }}\right)=Q^{-1}\left(p_{1}\right)=h_{1}$.

Observe that this approximation (which concentrates the available channel power onto one receiver) is equivalent to ideal receive beamforming followed by a single hard decision.

Similarly, $D\left(r_{\text {out }}\right)$ can be computed via the following steps:

1) Set $p_{1}=\cdots=p_{k}=p$ or, equivalently, $h_{1}=\cdots h_{k}=$ $h$. 
2) Solve $I_{\boldsymbol{h}}(X ; \boldsymbol{Z})=r_{\text {out }}$ to determine $p$ or $h=Q^{-1}(p)$.

3) Compute $D\left(r_{\text {out }}\right)=\sqrt{k} Q^{-1}(p)=\sqrt{k} h$.

This approximation amounts to setting the channel gains for all receivers to be equal (and applying hard decisions at each receiver prior to information combining). Intuitively, given a channel strength budget $\|\boldsymbol{h}\|$ for distributed reception with hard decision exchanges, concentrating all of the channel strength onto one receiver should provide better performance than dispersing it across all receivers evenly.

Based on the two-receiver results, we have

$$
p_{\text {out }}^{\mathrm{b} \rightarrow \text { hd }} \approx p_{k}\left(\alpha, C\left(r_{\text {out }}\right)\right) \approx p_{k}\left(\alpha, D\left(r_{\text {out }}\right)\right)
$$

for $k$ receivers with $p_{\text {out }}^{\mathrm{b} \rightarrow \text { hd }}=\operatorname{Prob}\left(I_{\boldsymbol{h}}(X ; \boldsymbol{Z})<r_{\text {out }}\right)$ and $p_{k}(\alpha, c)$ defined in (3).

To provide numerical evidence in support of the approximations, Fig. 3 shows the empirical distributions of the mutual information for distributed reception with hard decision exchanges for the case with i.i.d. Rayleigh channels normalized to $\|\boldsymbol{h}\|=C\left(r_{\text {out }}\right)$ and $\|\boldsymbol{h}\|=D\left(r_{\text {out }}\right)$ and $r_{\text {out }}=0.5$. For each $k \in\{2,5,10,20\}, 5000$ independent channel realizations were generated and the mutual information of each normalized channel realization was computed. These results show that the distribution of the mutual information of distributed reception with hard decision exchanges with channels on the outer radius $D\left(r_{\text {out }}\right)$ tends to be quite close to the actual outage rate $r_{\text {out }}=0.5$. Hence, at least in these examples, $p_{k}\left(\alpha, D\left(r_{\text {out }}\right)\right)$ is likely to be a better approximation than $p_{k}\left(\alpha, C\left(r_{\text {out }}\right)\right)$. This is also corroborated by the results in Section IV.
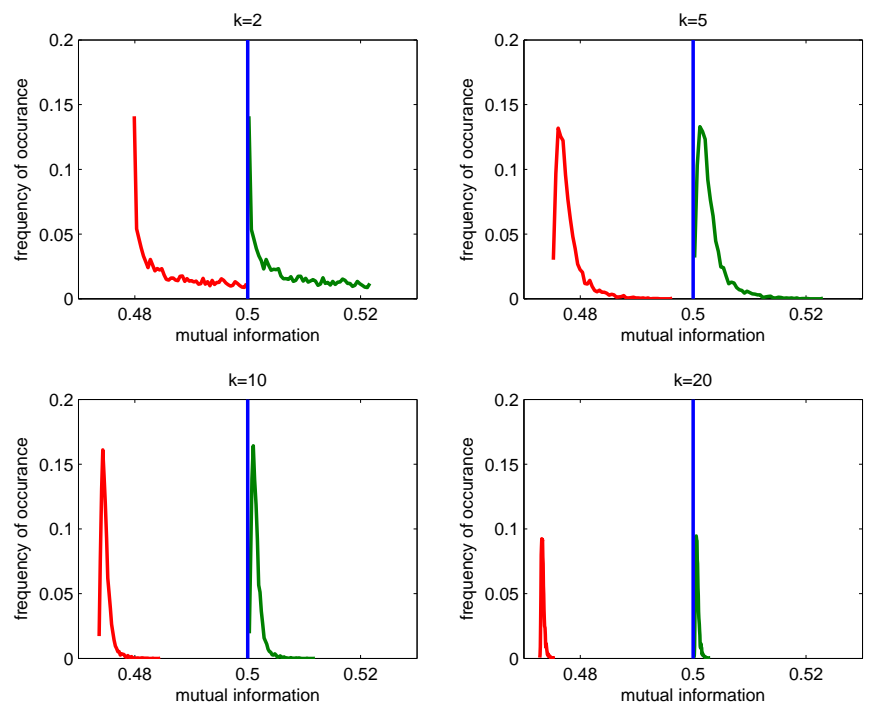

Fig. 3. Empirical distributions of $I_{\boldsymbol{h}}(X ; \boldsymbol{Z})$ for distributed reception with hard decision exchanges with normalized channels and outage rate $r_{\text {out }}=$ 0.5. The red and green curves show $I_{\boldsymbol{h}}(X ; \boldsymbol{Z})$ on the radii $\|\boldsymbol{h}\|=C\left(r_{\text {out }}\right)$ and $\|\boldsymbol{h}\|=D\left(r_{\text {out }}\right)$, respectively. The blue line is the outage rate

\section{NumericAl Results}

This section provides numerical results to illustrate the effect of $r_{\text {out }}$ and $k$ on the radii of the outage regions and the outage probability of distributed reception. Fig. 4 shows the four $\|\boldsymbol{h}\|$ radii developed in Section III as a function of $r_{\text {out }}$ for a $k=5$ receiver system. The radius $A\left(r_{\text {out }}\right)$ was computed explicitly from (7) and the remaining radii were computed using implicit function solvers via the procedures outlined in Section III. These results show that the inner and outer radii on the outage region of distributed reception with hard decision exchanges tend to be close unless $r_{\text {out }} \rightarrow 1$. These results also show that the outage regions for ideal beamforming with binary and Gaussian inputs tend to be close unless $r_{\text {out }} \rightarrow 1$.

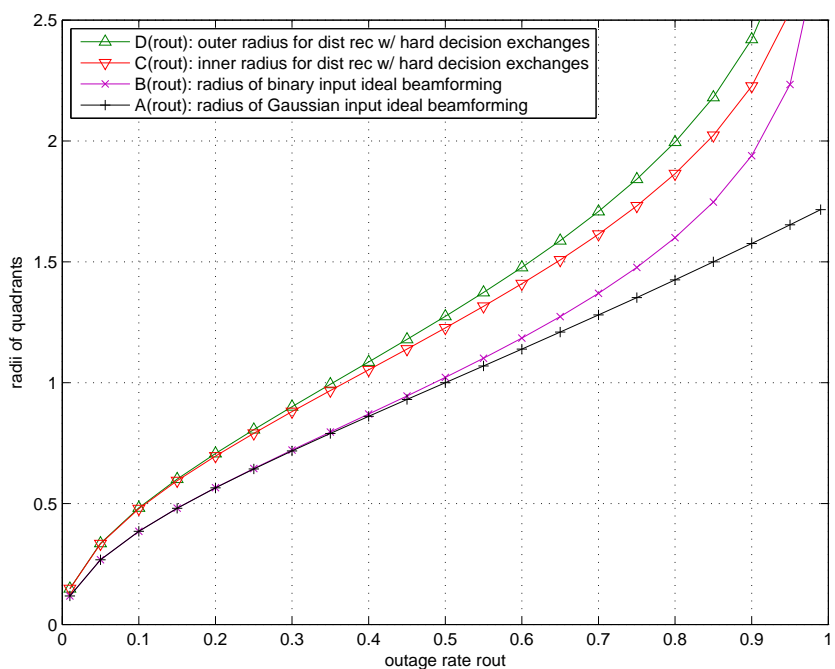

Fig. 4. Radii of outage quadrants as a function of $r_{\text {out }}$ for a $k=5$ receiver distributed reception system.

Note that an approximation for the performance gap in $\mathrm{dB}$ between ideal receive beamforming and distributed reception with hard decision exchanges can be computed by calculating $\beta\left(r_{\text {out }}\right)=20 \log _{10}\left(D\left(r_{\text {out }}\right) / B\left(r_{\text {out }}\right)\right)$ Fig. 5 plots this gap for $k=2, \ldots, 20$ and $r_{\text {out }} \in\{0.1,0.25,0.5,0.75,0.9\}$. These results show that the performance gap is always slightly less than $2 \mathrm{~dB}$ and appears to converge as $k \rightarrow \infty$ to a value close to the classic hard decision penalty of $10 \log _{10}(\pi / 2) \approx$ $1.96 \mathrm{~dB}$ [15]-[17] for the $r_{\text {out }}$ values tested.

Fig. 6 shows an outage probability simulation with outage probability plotted versus $\mathcal{E}_{s} / N_{0}$ for $k=1,2,5,10$ for a fixed outage rate $r_{\text {out }}=0.5$. The outage probabilities were computed over $10^{5}$ independent channel realizations with $g_{i} \stackrel{\text { i.i.d. }}{\sim} \mathcal{C N}(0,1)$. These results show that the outage probability of distributed reception with hard decision exchanges is well-approximated by the analysis in SectionIII and that the actual outage probability tends to be quite close to $p_{k}\left(\alpha, D\left(r_{\text {out }}\right)\right)$ corresponding to the outer integration region. The approximation resulting from the inner integration region $p_{k}\left(\alpha, C\left(r_{\text {out }}\right)\right)$ tends to be somewhat loose, especially for larger values of $k$. The gap between ideal receive beamforming and distributed reception with hard decision exchanges is consistent with Fig. 5. Note that the results reported in [14] tend to be somewhat better than those shown in Fig. 6, especially at smaller values of $k$, due to the fact that the distributed reception technique in [14] combines locally unquantized 


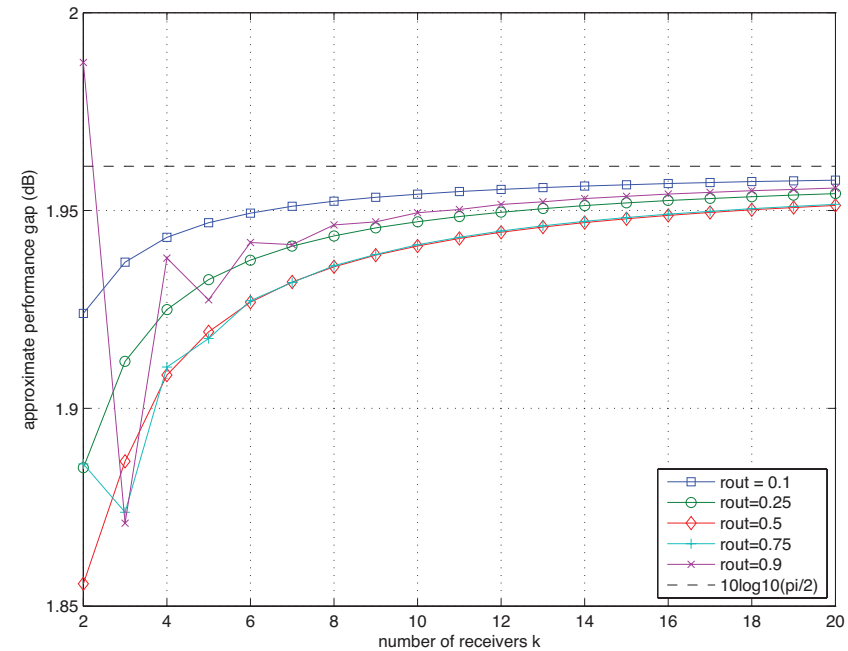

Fig. 5. Approximate performance gap in $\mathrm{dB}$ between ideal receive beamforming and distributed reception with hard decision exchanges versus $k$ and $r_{\text {out }}$.

information with the hard decisions from other receive nodes.

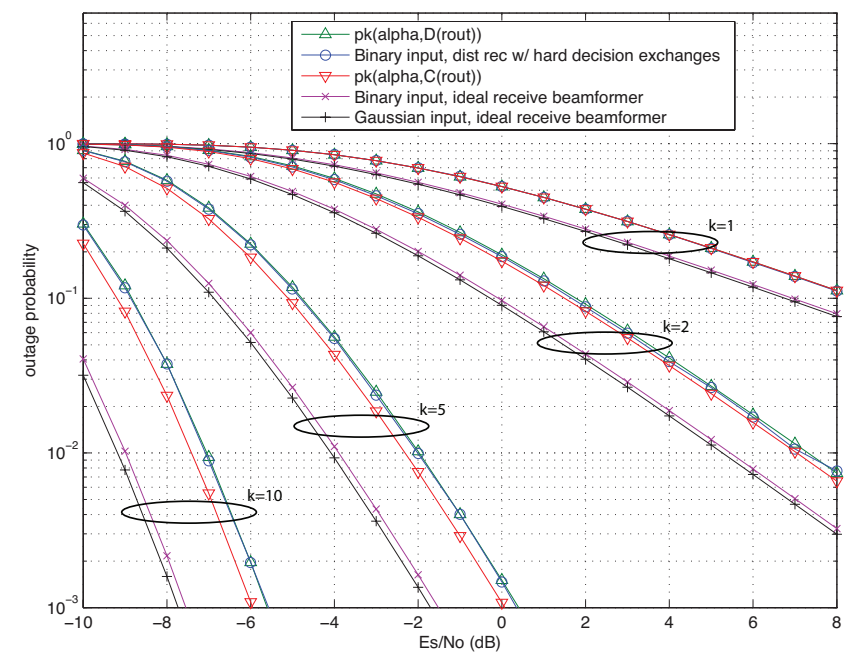

Fig. 6. Outage probability versus $\mathcal{E}_{s} / N_{0}$ and $k$ for ideal receive beamforming and distributed reception with hard decision exchanges with outage rate $r_{\text {out }}=0.5$.

While hard decisions exchanges add a severe nonlinearity to the receiver processing, these numerical results show that the performance of distributed reception with hard decision exchanges is still mainly determined by the channel norm $\|\boldsymbol{h}\|$. In fact, in Fig. 6, the actual outage probability of distributed reception with hard decision exchanges is almost indistinguishable from the approximation $p_{k}\left(\alpha, D\left(r_{\text {out }}\right)\right)$.

\section{CONCLUSION}

The numerical results in this paper indicate that the performance of distributed reception with binary hard decision exchanges is mainly governed by the SNR obtained by ideal receive beamforming (which is proportional to the square of the norm of the vector channel to the receivers), except for a performance loss of a little less than $2 \mathrm{~dB}$. For a given vector channel norm $\|\boldsymbol{h}\|$, concentrating the channel strength on one receiver gives an optimistic approximation for performance, while distributing the channel strength equally tends to give a slightly pessimistic approximation which is often close to the actual outage probability performance. We conjecture that these approximations actually bound the performance with hard decision exchanges, but are unable to provide a proof. Finding a proof or counterexample is an important direction for future work. Another potentially interesting extension of this work is to extend the analysis to higher-order forward link constellations, e.g., QPSK, 8PSK and 16-QAM.

\section{REFERENCES}

[1] G. Kramer, M. Gastpar, and P. Gupta, "Cooperative strategies and capacity theorems for relay networks," Information Theory, IEEE Transactions on, vol. 51, no. 9, pp. 3037 - 3063, September 2005.

[2] E. Aktas, J. Evans, and S. Hanly, "Distributed decoding in a cellular multiple-access channel," Wireless Communications, IEEE Transactions on, vol. 7, no. 1, pp. 241 -250, Janurary 2008.

[3] A. Sanderovich, S. Shamai, Y. Steinberg, and G. Kramer, "Communication via decentralized processing," Information Theory, IEEE Transactions on, vol. 54, no. 7, pp. 3008-3023, July 2008

[4] I.-H. Wang and D. Tse, "Interference mitigation through limited receiver cooperation: Symmetric case," in Information Theory Workshop, 2009. ITW 2009. IEEE, October 2009, pp. 579 -583.

[5] S. Yi, B. Azimi-Sadjadit, S. Kalyanaraman, and V. Subramanian, "Error control code combining techniques in cluster-based cooperative wireless networks," in Communications, 2005. ICC 2005. 2005 IEEE International Conference on, vol. 5, May 2005, pp. 3510 - 3514 Vol. 5.

[6] S. Yi, S. Kalyanaraman, B. Azimi-Sadjadi, and H. Shen, "Energyefficient cluster-based cooperative fec in wireless networks," Wireless Networks, vol. 15, no. 8, pp. 965-977, November 2009.

[7] J. Choi and S. Choi, "Cooperative and distributed receiver processing based on message passing," Vehicular Technology, IEEE Transactions on, vol. 60, no. 7, pp. 3066 -3075, September 2011.

[8] T. Wong, X. Li, and J. Shea, "Distributed decoding of rectangular paritycheck code," Electronics Letters, vol. 38, no. 22, pp. 1364 - 1365, October 2002.

[9] - "Iterative decoding in a two-node distributed array," in MILCOM 2002. Proceedings, vol. 2, October 2002, pp. 1320 - 1324 vol.2.

[10] X. Li, T. Wong, and J. Shea, "Bit-interleaved rectangular parity-check coded modulation with iterative demodulation in a two-node distributed array," in Communications, 2003. ICC '03. IEEE International Conference on, vol. 4, May 2003, pp. 2812 - 2816 vol.4.

[11] A. Avudainayagam, J. Shea, T. Wong, and X. Li, "Reliability exchange schemes for iterative packet combining in distributed arrays," in Wireless Communications and Networking, 2003. WCNC 2003. 2003 IEEE, vol. 2, March 2003, pp. $832-837$ vol.2.

[12] A. Nayagam, J. Shea, and T. Wong, "Collaborative decoding in bandwidth-constrained environments," Selected Areas in Communications, IEEE Journal on, vol. 25, no. 2, pp. 434 -446, February 2007.

[13] X. Li, T. Wong, and J. Shea, "Performance analysis for collaborative decoding with least-reliable-bits exchange on awgn channels," Сотmunications, IEEE Transactions on, vol. 56, no. 1, pp. $58-69$, January 2008.

[14] D. R. Brown III, M. Ni, U. Madhow, and P. Bidigare, "Distributed reception with coarsely-quantized observation exchanges," in Information Sciences and Systems, 2013. CISS 2013. 47th Annual Conference on, Mar 20-22. 2013.

[15] T. Tozer and J. Kollerstrom, "Penalties of hard decision in signal detection," Electronics Letters, vol. 16, no. 5, pp. 199-200, 1980.

[16] N. Beaulieu and C. Leung, "On the performance of three suboptimum detection schemes for binary signaling," Communications, IEEE Transactions on, vol. 33, no. 3, pp. 241-245, 1985.

[17] D. R. Brown III, U. Madhow, M. Ni, M. Rebholz, and P. Bidigare, "Distributed reception with hard decision exchanges," Submitted to IEEE Transactions on Wireless Communications, in review.

[18] S. Haykin, Communication Systems, 5th ed. Wiley, 2009. 